BRIEF COMMUNICATION

\title{
Extracellular amoebal-vesicles: potential transmission vehicles for respiratory viruses
}

\author{
Rafik Dey $\mathbb{D}^{1,2 凶}$, Melanie A. Folkins $\mathbb{i D}^{2}$ and Nicholas J. Ashbolt $\mathbb{i D}^{1,2,3}$
}

Human respiratory syncytial virus (RSV) is a major cause of acute respiratory tract infections in children and immunocompromised adults worldwide. Here we report that amoebae-release respirable-sized vesicles containing high concentrations of infectious RSV that persisted for the duration of the experiment. Given the ubiquity of amoebae in moist environments, our results suggest that extracellular amoebal-vesicles could contribute to the environmental persistence of respiratory viruses, including potential resistance to disinfection processes and thereby offering novel pathways for viral dissemination and transmission.

npj Biofilms and Microbiomes (2021)7:25; https://doi.org/10.1038/s41522-021-00201-y

Amoebae are amongst the most ubiquitous organisms in natural and engineered environments ${ }^{1-3}$. They live at interfaces (water-soil, water-animal, water-plants and water-air), adherent on various surfaces and feed on microorganisms ${ }^{3}$. While relatively few amoebae species are pathogens in their own right ${ }^{4}$, they are known natural environmental reservoirs for a range of amoeba-resisting bacterial pathogens, such as Legionella pneumophila, a water-based bacterium responsible for Legionnaires' disease that results in major community health burden ${ }^{5-8}$. More recently, amoebae have been identified as potential reservoirs for non-enveloped respiratory and enteric viruses such as adenoviruses, coxsackieviruses, reovirus and the giant amoeba virus Mimivirus ${ }^{9-12}$. Several highly transmissible respiratory enveloped viruses with epidemic potential have emerged in last two decades, with the ongoing COVID-19 pandemic being the most significant to date ${ }^{13}$, yet their potential interaction with (sewage/faecal-borne) amoebae is unreported.

Human respiratory syncytial virus (RSV) is a large $(120-300 \mathrm{~nm}$ diameter) pleomorphic enveloped virus with a non-segmented, negative-sense, single-stranded RNA that belongs to the Pneumoviridae family and is recognised as one of the most common causes of acute respiratory tract infections in children, older people, and immunocompromised adults ${ }^{14-16}$. Despite the enormous burden of RSV disease, there is currently no efficacious vaccine nor antiviral drug therapy available ${ }^{17}$. RSV is a highly contagious pathogen and transmission is thought to be primarily by large droplets and fomites, but is yet to be fully resolved ${ }^{18}$. However, clinical and epidemiological studies of patients infected with RSV raised the possibility of faecal-oral transmission as described for other respiratory viruses ${ }^{19-21}$. Herein we used RSV as a model for potential interactions of enveloped respiratory viruses with amoebae to ascertain their possible role as an environmental reservoir and vehicle for dissemination and transmission.

Within two hours of introducing GFP-RSV to an active culture of Willaertia magna (co-culture) the virus was observed within trophozoites and expelled vesicles (Fig. 1a). In a separate experiment, and after $72 \mathrm{~h}$ post introduction, fluorescence microscopy showed expelled respirable-sized amoebal-vesicles filled with GFP-RSV (Fig. 1b). Transmission electron microscopy (TEM) revealed pleomorphic RSV particles from different crosssections within W. magna phagosomes (Fig. 2a). Further to this, the presence of RSV inside purified extracellular amoebal-vesicles was confirmed by TEM (Fig. 2b). Using the ImageJ software package ${ }^{22}$, the virions measurements (Table 1) were consistent with previous conventional EM studies ${ }^{23-25}$.

It is important to note, that amoebae trophozoites were visibly unaffected by the presence of internalised RSV virus.

Based on the GFP expression, it appeared that the RSV within amoebal-vesicles could still be infectious ${ }^{26,27}$. Therefore, it was of interest to assess the infectivity of freshly isolated RSV-EAVs (Fig. 3a). The EAVs containing RSV were collected $24 \mathrm{~h}$ post infection and viral titres, as measured by traditional $\mathrm{TCID}_{50}$ analysis, demonstrated that RSV-EAVs were indeed infectious with titres peaking at $\sim 10^{4} \mathrm{TCID}_{50} \mathrm{~mL}^{-1}$ (Fig. 3C), at a similar infectivity to RSV-only controls. Minor losses could be explained by the supernatant washing steps. On closer observation using phasecontrast microscopy there was also clear cytopathic effect induced by infectious RSV-EAVs in Hela cells, preventing the formation of the cells monolayer and affecting their appearance after 5 days of infection (Fig. 3d).

Recently, multiple independent studies have revealed that different viruses may exploit the secretory autophagy pathway to exit cells via released vesicles ${ }^{28-31}$. These amoebal-released packaged viruses could prolong their environmental infectivity (via fomites/aerosols/water system), as well when internalised by avoiding immune systems detection, such as evading recognition by neutralising antibodies ${ }^{32}$. Also, in a previous study utilising infectious Coxsackievirus B virions (i.e. a non-enveloped, enteric virus) we reported virions localised in Vermamoeba vermiformis trophozoites and expelled vesicles ${ }^{11}$. Overall, virus-laden vesicles would increase the (dose) likelihood to infect susceptible host cells $^{33}$, as well as the virus' infectivity, as demonstrated for enteroviruses with equivalent numbers of virions free versus within vesicles $29,34,35$. Extracellular vesicles containing enteric viruses are naturally shed in human and animal faeces (and amoebae grow in sewage/animal excreta, including bat guano $)^{36-38}$, which could be ingested and transmit to other hosts ${ }^{39}$. Interestingly, as evident in Figs 1 and 2, the released amoebal-vesicles are 2-3 $\mu \mathrm{m}$ in diameter, the size range expected to penetrate to the lower respiratory tract via mouth or nose inhalation ${ }^{40,41}$.

Taken together these interesting observations provide evidence to suggest that amoebae may contribute to the environmental persistence and transmission of respiratory viruses associated with

${ }^{1}$ School of Public Health, University of Alberta, Edmonton, AB, Canada. ${ }^{2}$ Dept. Medical Microbiology and Immunology, University of Alberta, Edmonton, AB, Canada. ${ }^{3}$ School of Environment, Science and Engineering, Southern Cross University, Lismore, NSW, Australia. ${ }_{\text {email: rafik@ualberta.ca }}$ 

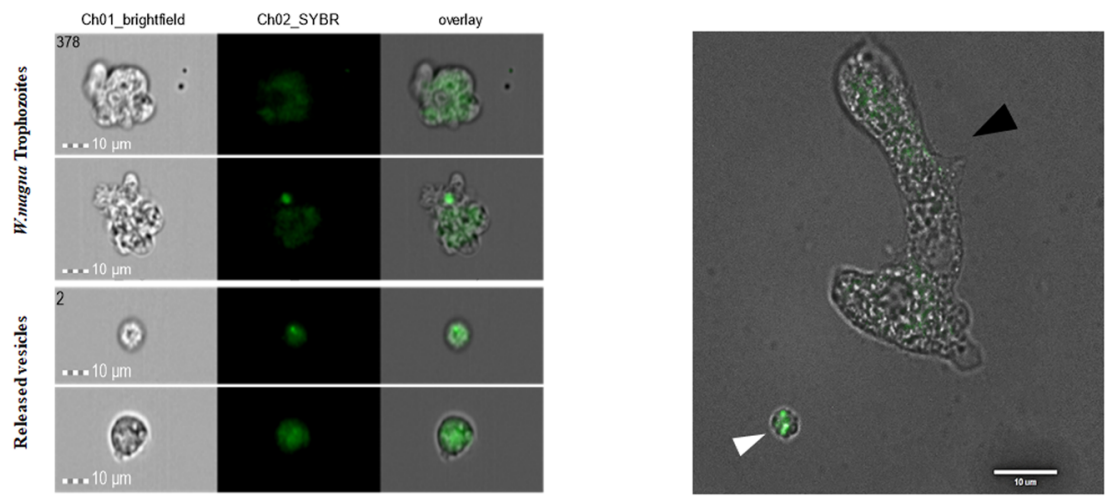

Fig. 1 Intracellular RSV localisation. a Internalised GFP-RSV virus particles in live amoebae (trophozoites and released vesicles) using ImageStream flow cytometry. b GFP-RSV virus particles packaged in excreted vesicles by W. magna using fluorescent microscopy (100x), released vesicle (white arrowhead) amoeba trophozoites (black arrowhead).
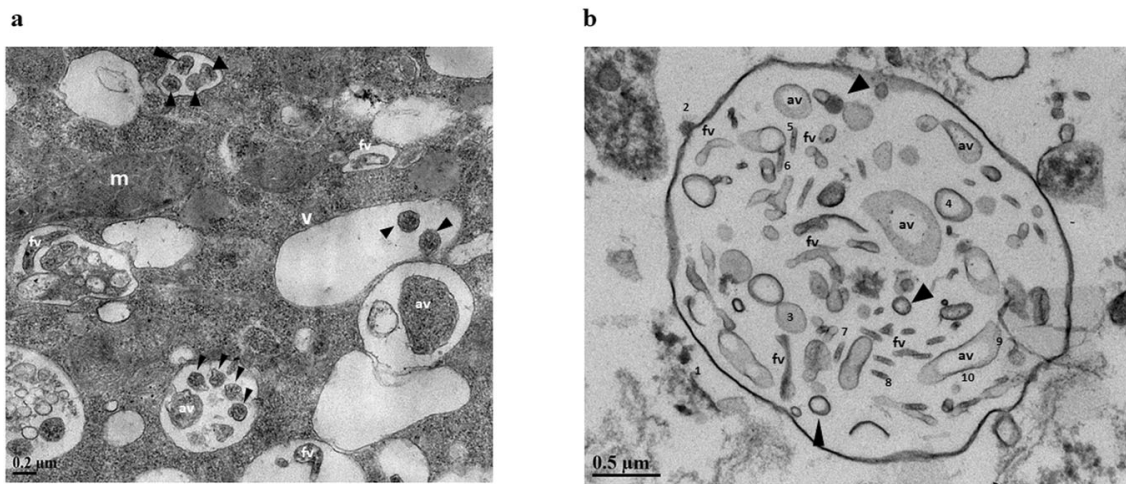

Fig. 2 Ultrastructural visualisation of internalised RSV. a Transmission electron microscopy of RSV particles within W. magna food vacuoles after $72 \mathrm{~h}$ of co-culture. b Purified released extracellular vesicle after $24 \mathrm{~h}$ of co-culture containing RSV virions. Virions were randomly selected and measured using ImageJ (marked 3-10). Three morphology categories of RSV were found: spherical (black arrowheads), asymmetric (marked av), and filamentous (marked fv). mitochondria (m), vacuole (v).

Table 1. Extracellular Amoebal Vesicle (EAV) and internalised RSV virion measurements.

\begin{tabular}{|c|c|c|c|c|c|c|c|}
\hline & Area & Mean & Min & Max & Angle & Length $\mathrm{nm}$ & Shape \\
\hline 1 (width) & 10344.39 & 172.874 & 24.109 & 240.099 & 0.141 & 2892.866 & Spherical \\
\hline \multicolumn{8}{|l|}{ RSV virions } \\
\hline 3 & 688.776 & 160.64 & 60 & 206.963 & -128.83 & 187.967 & Spherical \\
\hline 6 & 165.816 & 93.058 & 73.75 & 126 & -4.764 & 43.006 & Filamentous \\
\hline 7 & 178.571 & 129.304 & 96.314 & 182.515 & -22.62 & 46.429 & Filamentous \\
\hline 8 & 165.816 & 114.662 & 92 & 150 & -109.983 & 41.802 & Filamentous \\
\hline 9 & 1785.714 & 185.194 & 125 & 229.638 & 28.775 & 497.096 & Asymmetric \\
\hline 10 & 816.327 & 171.882 & 102.797 & 208.049 & -61.39 & 223.749 & Asymmetric \\
\hline
\end{tabular}

natural aquatic environments and engineered water systems. Notably, extracellular amoebal-vesicles could enable nonenveloped and enveloped virion dissemination and aid in the transmission of respiratory viruses. Amoeba-packaged viruses (in trophozoites, cysts and vesicles) may also protect virions from inactivation via sunlight, biocides ${ }^{42}$ and antiviral host factors ${ }^{43,44}$. Hence, we recommend further study of the persistence and transmission of respiratory viruses in faecal droplets and aerosols to assess this newly proposed risk pathway; noting that sewage droplets/aerosols were shown to be important during the first SARS epidemic ${ }^{45}$, and associated with toilets and COVID-19 cases in hospitals ${ }^{46}$. Understanding how enveloped viruses persists in our environmental systems and interact with amoebae will contribute to our understanding of the epidemiology and 
a

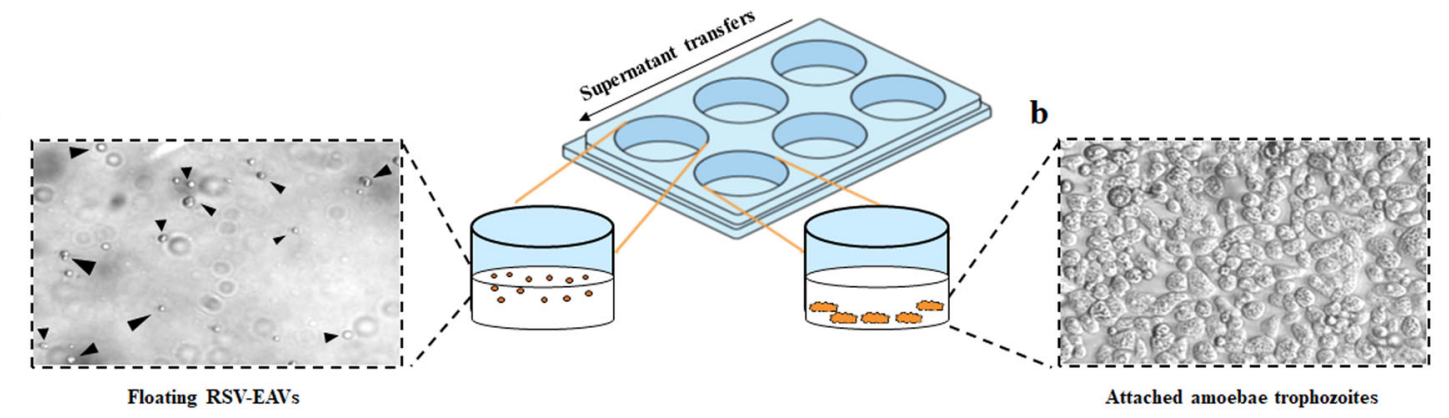

c

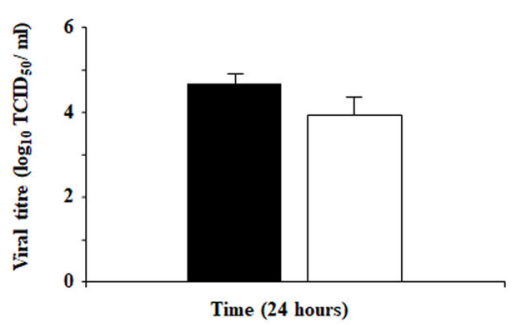

d

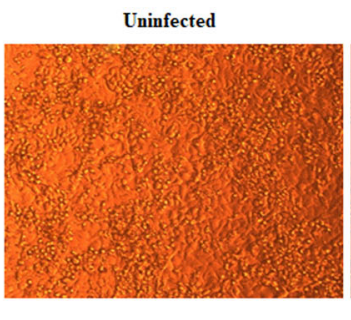

Infected with RSV only

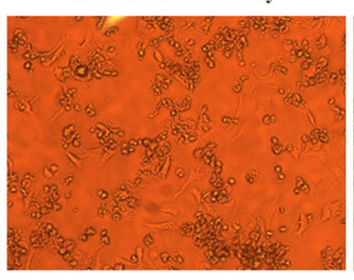

Infected with RSV-EAVs

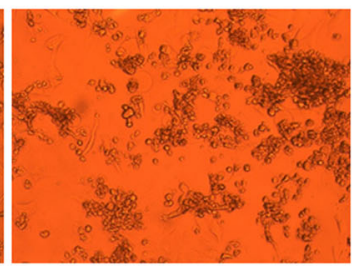

Fig. 3 RSV-EAVs isolation and infectivity assay. Micrographs (40x) of a Released extracellular amoebal-vesicles containing RSV and b Attached amoebae trophozoites. c Replication kinetics of the control RSV alone (black bar) and RSV-EAVs (white bar) analysed by TCID50 on Hela cells. Data are the mean \pm SEM, $n=3$ performed in triplicate. d Micrographs (20x) of cytopathic effect (CPE) induced by RSV-EAVs infection in Hela cells at 5 days post-infection.

microbial ecology of respiratory viruses and potentially permit the development of methods to further aid in their management.

\section{METHODS}

\section{Strains and culture conditions}

The virus used in this study was green fluorescent protein-expressing RSV (GFP-RSV) containing the viral glycoproteins $(\mathrm{S}, \mathrm{G} \text { and } \mathrm{F})^{47}$. The RSV was propagated on $80-90 \%$ confluent HeLa cells (ATCC CCL-2) in DMEM medium containing $10 \% \mathrm{FBS}$, and $1 \%$ penicillin-streptomycin at $37^{\circ} \mathrm{C}$ and $5 \% \mathrm{CO}_{2}$ in vented $75 \mathrm{~cm}^{2}$ cell-culture flasks.

The amoebae used in this study was Willaertia magna (ATCC 50035), a member of the Vahlkampfiidae family that was isolated from bovine faeces. Amoebae were grown in tissue culture flasks in SCGYEM (SerumCasein-Glucose-Yeast-Extract-Medium: ATCC medium 1021) at $25^{\circ} \mathrm{C}$ in a $5 \% \mathrm{CO}_{2}$ incubator. The trophozoites were maintained in exponential growth phase by sub-culturing every 3-4 days in fresh SCGYEM. Amoebae were harvested by tapping the flasks to dislodge surface-adhered cells and subsequent centrifugation in a $15 \mathrm{~mL}$ screw-cap tube (FALCON, Fischer Scientific, Edmonton, Canada 3033) at $2000 \times g$ for $10 \mathrm{~min}$. Cells were washed three times with sterile distilled water to remove carried-over nutrients in the supernatants.

\section{Imaging flow cytometry analysis}

ImageStream cytometry analysis and the instrument gating strategy for amoebae was performed as previously described ${ }^{37}$. Briefly, W. magna trophozoites were infected for $2 \mathrm{~h}$ with GFP-RSV at MOI of 100, washed and re-suspended in PBS prior to processing through the ImageStream $\mathrm{X}$ Mark II (Millipore Sigma). Cells were examined at $60 \times$ magnification. Analysis was performed using the IDEAS software (Amnis, Seattle) and cells (fluorescent viruses and amoebae) were identified on the basis of bright field morphology, size and GFP signal.

\section{Isolation of extracellular amoebal-vesicles (EAVs) containing RSV}

W. magna and RSV were co-cultured at a ratio of 1:100 in conical Falcon tubes containing $3 \mathrm{~mL}$ of SCGYEM medium, vortexed to favour virus interaction with amoebae and then transferred to 6-well culture plates (Fisher Scientific 130185). After overnight incubation at $30^{\circ} \mathrm{C}$, samples were analysed using a phase-contrast microscope (Leica CTR 4000) to detect the presence of EAVs in the supernatant while amoebal trophozoites remain attached to the surface of the well plates. To isolate and separate the EAVs containing RSV from the attached trophozoites, supernatants were removed and transferred into new well plates several times. In brief, supernatants were gently removed with care taken not to disturb the attached amoebae on well plate surfaces, and transferred to new well plates for 10-20 min to allow any amoebal trophozoites to attach to surfaces (Fig. 3b). The isolated EAVs containing RSV were collected and washed twice with PBS by centrifugation at $4000 \times g$ for 5 min to remove uninternalized viruses. The purified EAVs were then used for infectivity assays and microscopy.

\section{RSV infectivity assays}

RSV was released from amoebal vesicles by three consecutive freeze-thaw cycles. RSV infectivity (EAVs containing RSV and RSV-only control) was measured by infecting confluent HeLa cells in quadruplicate using 48-well plates and serial dilution of the virus in HeLa cells maintenance medium. Cells were observed daily for cytopathic effects for seven days and CPE was measured by the tissue culture infectious dose $50 \%\left(\mathrm{TCID}_{50}\right)$ using the Reed-Muench formula ${ }^{48}$.

\section{Transmission electron microscopy}

Axenic cultures of W. magna were co-cultured with RSV at a MOI of 100 on Thermonax cover slips (Thermo Fisher 174985). After decanting the medium, amoebae were fixed at room temperature with $2.5 \%$ glutaraldehyde and $0.1 \mathrm{M}$ sodium cacodylate buffer (Electron Microscopy Sciences 15960). The samples were submitted for processing at the imaging core at University of Alberta, faculty of biological sciences. Sectioned and carbon-coated samples were observed with a Hitachi $\mathrm{H}$ 7650 transmission electron microscope.

\section{Fluorescence microscopy}

Co-cultures of W. magna-GFP-RSV were carried in 12-well tissue culture plates overlaid with microscopy cover slips (Fisher Scientific 12-5461) and 
incubated at $25^{\circ} \mathrm{C}$ with $5 \% \mathrm{CO}_{2}$. After $72 \mathrm{~h}$ of infection, the medium was removed and cells fixed with $4 \%$ paraformaldehyde for $5 \mathrm{~min}$ at room temperature and then washed with phosphate-buffered saline three times. Images were taken with an EVOS FL fluorescent cell imaging system (ThermoFisher Scientific).

\section{Reporting summary}

Further information on research design is available in the Nature Research Reporting Summary linked to this article.

\section{DATA AVAILABILITY}

The data sets generated during and/or analysed during the current study are either shown in the manuscript or available from the corresponding author on reasonable request.

Received: 17 September 2020; Accepted: 18 February 2021; Published online: 17 March 2021

\section{REFERENCES}

1. Thomas, V., Loret, J. F., Jousset, M. \& Greub, G. Biodiversity of amoebae and amoebae-resisting bacteria in a drinking water treatment plant. Environ. Microbiol. 10, 2728-2745 (2008)

2. Ashbolt, N. J. Microbial contamination of drinking water and human health from community water systems. Curr. Environ. Health Rep. 2, 95-106 (2015).

3. Rodriguez-Zaragoza, S. Ecology of free-living amoebae. Crit. Rev. Microbiol 20, 225-241 (1994)

4. Abdul Majid, M. A. et al. Pathogenic waterborne free-living amoebae: an update from selected Southeast Asian countries. PLoS One 12, e0169448 (2017).

5. Rowbotham, T. J. Current views on the relationships between amoebae, legionellae and man. Isr. J. Med Sci. 22, 678-689 (1986).

6. Cunha, B. A., Burillo, A. \& Bouza, E. Legionnaires' disease. Lancet 387, 376-385 (2016).

7. Molmeret, M., Horn, M., Wagner, M., Santic, M. \& Abu Kwaik, Y. Amoebae as training grounds for intracellular bacterial pathogens. Appl Environ. Microbiol 71, 20-28 (2005).

8. Cassini, A. et al. Impact of infectious diseases on population health using incidence-based disability-adjusted life years (DALYs): results from the burden of communicable diseases in Europe study, European Union and European economic area countries, 2009 to 2013. Eur. Surveill. 23, https://doi.org/10.2807/15607917.ES.2018.23.16.17-00454 (2018).

9. La Scola, B., Marrie, T. J., Auffray, J. P. \& Raoult, D. Mimivirus in pneumonia patients. Emerg. Infect. Dis. 11, 449-452 (2005).

10. Scheid, P. \& Schwarzenberger, R. Acanthamoeba spp. as vehicle and reservoir of adenoviruses. Parasitol. Res. 111, 479-485 (2012).

11. Atanasova, N. D. et al. Persistence of infectious enterovirus within free-living amoebae - a novel waterborne risk pathway? Water Res. 144, 204-214 (2018).

12. Folkins, M. A., Dey, R. \& Ashbolt, N. J. Interactions between human reovirus and free-Living amoebae: implications for enteric virus disinfection and aquatic persistence. Environ. Sci. Technol. 54, 10201-10206 (2020).

13. Bayham, J. \& Fenichel, E. P. Impact of school closures for COVID-19 on the US health-care workforce and net mortality: a modelling study. Lancet Public Health, https://doi.org/10.1016/S2468-2667(20)30082-7 (2020).

14. Nam, H. H. \& Ison, M. G. Respiratory syncytial virus infection in adults. BMJ 366, 15021 (2019).

15. Haber, N. Respiratory syncytial virus infection in elderly adults. Med Mal. Infect. 48, 377-382 (2018)

16. Simonsen, L. \& Viboud, C. Respiratory syncytial virus infection in elderly adults. $N$. Engl. J. Med 353, 422-423 (2005). author reply 422-423.

17. Collins, P. L. \& Graham, B. S. Viral and host factors in human respiratory syncytial virus pathogenesis. J. Virol. 82, 2040-2055 (2008).

18. Drysdale, S. B., Sande, C. J., Green, C. A. \& Pollard, A. J. RSV vaccine use-the missing data. Expert Rev. Vaccines 15, 149-152 (2016).

19. Zhu, Z. et al. Extra-pulmonary viral shedding in H7N9 avian influenza patients. J. Clin. Virol. 69, 30-32 (2015).

20. Chan, M. C. et al. Seasonal influenza A virus in feces of hospitalized adults. Emerg. Infect. Dis. 17, 2038-2042 (2011).

21. von Linstow, M. L. et al. Excretion patterns of human metapneumovirus and respiratory syncytial virus among young children. Eur. J. Med. Res. 11, 329-335 (2006).
22. Schneider, C. A., Rasband, W. S. \& Eliceiri, K. W. NIH image to imageJ: 25 years of image analysis. Nat. Methods 9, 671-675 (2012).

23. Bachi, T. \& Howe, C. Morphogenesis and ultrastructure of respiratory syncytial virus. J. Virol. 12, 1173-1180 (1973)

24. Bajorek, M. et al. The Thr205 phosphorylation site within respiratory syncytial virus matrix $(\mathrm{M})$ protein modulates $\mathrm{M}$ oligomerization and virus production. J. Virol. 88, 6380-6393 (2014).

25. Liljeroos, L., Krzyzaniak, M. A., Helenius, A. \& Butcher, S. J. Architecture of respiratory syncytial virus revealed by electron cryotomography. Proc. Natl Acad. Sci. USA 110, 11133-11138 (2013).

26. Sastre, P., Oomens, A. G. \& Wertz, G. W. The stability of human respiratory syncytial virus is enhanced by incorporation of the baculovirus GP64 protein. Vaccine 25, 5025-5033 (2007).

27. Oomens, A. G., Megaw, A. G. \& Wertz, G. W. Infectivity of a human respiratory syncytial virus lacking the $\mathrm{SH}, \mathrm{G}$, and $\mathrm{F}$ proteins is efficiently mediated by the vesicular stomatitis virus G protein. J. Virol. 77, 3785-3798 (2003).

28. Robinson, S. M. et al. Coxsackievirus B exits the host cell in shed microvesicles displaying autophagosomal markers. PLoS Pathog. 10, e1004045 (2014).

29. Chen, Y. H. et al. Phosphatidylserine vesicles enable efficient en bloc transmission of enteroviruses. Cell 160, 619-630 (2015).

30. Bird, S. W., Maynard, N. D., Covert, M. W. \& Kirkegaard, K. Nonlytic viral spread enhanced by autophagy components. Proc. Natl Acad. Sci. USA 111, 13081-13086 (2014).

31. Zhang, Z. W., Li, Z. L. \& Yuan, S. The role of secretory autophagy in zika virus transfer through the placental barrier. Front Cell Infect. Microbiol. 6, 206 (2016).

32. Feng, Z. et al. A pathogenic picornavirus acquires an envelope by hijacking cellular membranes. Nature 496, 367-371 (2013).

33. Altan-Bonnet, N. \& Chen, Y. H. Intercellular transmission of viral populations with vesicles. J. Virol. 89, 12242-12244 (2015).

34. Mutsafi, Y. \& Altan-Bonnet, N. Enterovirus transmission by secretory autophagy. Viruses 10, https://doi.org/10.3390/v10030139 (2018).

35. Borderia, A. V. et al. Group selection and contribution of minority variants during virus adaptation determines virus fitness and phenotype. PLoS Pathog. 11, e1004838 (2015).

36. Garcia, A. et al. Potentially pathogenic free-living amoebae (FLA) isolated in Spanish wastewater treatment plants. Environ. Microbiol Rep. 3, 622-626 (2011).

37. Fernandez-Cassi, X. et al. Evaluation of the microbiological quality of reclaimed water produced from a lagooning system. Environ. Sci. Pollut. Res. Int. 23, 16816-16833 (2016)

38. Mulec, J., Dietersdorfer, E., Ustunturk-Onan, M. \& Walochnik, J. Acanthamoeba and other free-living amoebae in bat guano, an extreme habitat. Parasitol. Res. 115, 1375-1383 (2016).

39. Santiana, M. et al. Vesicle-cloaked virus clusters are optimal units for interorganismal viral transmission. Cell Host Microbe 24, 208-220 (2018). e208.

40. Swuste, P., Corn, M. \& Goelzer, B. Hazard prevention and control in the work environment. Report of a WHO meeting. Int J. Occup. Med. Environ. Health 8, 7-10 (1995).

41. Allegra, S. et al. Characterization of aerosols containing legionella generated upon nebulization. Sci. Rep. 6, 33998 (2016).

42. Boratto, P. V. et al. Amoebas as mimivirus bunkers: increased resistance to UV light, heat and chemical biocides when viruses are carried by amoeba hosts. Arch. Virol. 159, 1039-1043 (2014).

43. Altan-Bonnet, N. Extracellular vesicles are the trojan horses of viral infection. Curr. Opin. Microbiol 32, 77-81 (2016).

44. Altan-Bonnet, N., Perales, C. \& Domingo, E. Extracellular vesicles: vehicles of en bloc viral transmission. Virus Res. 265, 143-149 (2019).

45. Yu, I. T., Qiu, H., Tse, L. A. \& Wong, T. W. Severe acute respiratory syndrome beyond amoy gardens: completing the incomplete legacy. Clin. Infect. Dis. 58, 683-686 (2014).

46. Liu, Y. et al. Aerodynamic analysis of SARS-CoV-2 in two wuhan hospitals. Nature 582, 557-560 (2020).

47. Kwilas, S. et al. Respiratory syncytial virus grown in vero cells contains a truncated attachment protein that alters its infectivity and dependence on glycosaminoglycans. J. Virol. 83, 10710-10718 (2009).

48. Reed, L. J. \& Muench, H. A simple method of estimating fifty percent endpoints. Am. J. Trop. Med. Hyg. 27, 493-497 (1938).

\section{ACKNOWLEDGEMENTS}

These studies were supported by Alberta Innovates (grant \# 201300490), Alberta, Canada. We would like to thank Dr. David Marchant (University of Alberta; Canada) for supplying the green fluorescent protein-expressing RSV (GFP-RSV) strain used in this study and Arlene Oatway for help with transmission electron microscopy (Microscopy Facility Biological Sciences, University of Alberta). 


\section{AUTHOR CONTRIBUTIONS}

R.D. conceived the work. Sample preparations and assays were carried out by M.F. and R.D. R.D. and N.J.A wrote the paper.

\section{COMPETING INTERESTS}

The authors declare no competing interests.

\section{ADDITIONAL INFORMATION}

Supplementary information The online version contains supplementary material available at https://doi.org/10.1038/s41522-021-00201-y.

Correspondence and requests for materials should be addressed to R.D.

Reprints and permission information is available at http://www.nature.com/ reprints
Publisher's note Springer Nature remains neutral with regard to jurisdictional claims in published maps and institutional affiliations. (i) Open Access This article is licensed under a Creative Commons Attribution 4.0 International License, which permits use, sharing adaptation, distribution and reproduction in any medium or format, as long as you give appropriate credit to the original author(s) and the source, provide a link to the Creative Commons license, and indicate if changes were made. The images or other third party material in this article are included in the article's Creative Commons license, unless indicated otherwise in a credit line to the material. If material is not included in the article's Creative Commons license and your intended use is not permitted by statutory regulation or exceeds the permitted use, you will need to obtain permission directly from the copyright holder. To view a copy of this license, visit http://creativecommons. org/licenses/by/4.0/.

(c) The Author(s) 2021 